\title{
Mujeres del Desierto rompiendo estereotipos en \\ Ingeniería
}

\section{Desert's woman braking serotypes in engineering}

\author{
Guadalupe Jiménez Hidalgo ${ }^{1}$
}

\begin{abstract}
Resumen
El acceso de las mujeres a la educación debe ser una realidad, no solo un aspecto jurídico; ellas pueden inscribirse en cualquier licenciatura; existen carreras mayoritariamente con presencia de hombres donde existe segregación, se generan estereotipos y permiten la reproducción de los modelos de poder. Es importante en la mirada de los estudios de género, la historia individual de aquellas que hacen referencia en la vida cotidiana, a sus características que, las constituyen como mujeres fuera del estándar, por rebasar los estereotipos impuestos, listas a disminuir la segregación con su lucha, impulsando una movilidad social, combatiendo para eliminar la brecha salarial, cuando se atreven a estudiar carreras con una matrícula predominantemente de hombres. Las mujeres que estudian Ingeniería Mecatrónica e Ingeniería Electromecánica son un claro ejemplo de ello. Observar a estas alumnas permite generar elementos de autoreflexión, cuando mencionan, "Yo puedo cursar esta carrera que dicen: esta difícil y es de hombres". Las manifestaciones de los estereotipos para las estudiantes de ingeniería, son un reto y un punto de inflexión para cambiar las concepciones pre establecidas por su comunidad. Se les ve como Hombre, que no son capaces de hacer lo que los hombres hacen, que son feas, desarregladas, nerds, marimachas; estas mujeres, por el contrario, se perciben unas a otras como mujeres que se maquillan, usan vestidos, tacones, van de fiesta, son inteligentes y son capaces de hacer lo que quieren como cualquier ser humano, sin importar el qué dirán, rompiendo en el desierto los estereotipos de las ingenierías.
\end{abstract}

\footnotetext{
1 Guadalupe Jiménez Hidalgo. Profesora del TecNM: Instituto Tecnológico de Ciudad Jiménez, Chihuahua, México. Es Maestra en Administración y tiene publicaciones CICA 2016, CICA 2017, III Congreso Investigación Educativa en el Estado de Chihuahua. Es integrante del cuerpo académico de Innovación Educativa y Matemáticas. Correo electrónico: gpejimenezh@yahoo.com

ID: http://orcid.org/0000-0002-8257-3093
} 
RECIE. Revista Electrónica Científica de Investigación Educativa Vol. 4, núm. 2, enero-diciembre 2019, pp. 1281-1290.

\title{
Palabras clave \\ Mujeres, estudios de género, estereotipos, ingeniería.
}

\begin{abstract}
The access to education for women needs to be a reality, not just a law, finally women can enroll in any career, nevertheless there are still degrees domineered by the majority by man in which segregation still exist, and stereotypes arise letting the power models to spread. It is important in the perspective of gender studies, the individual history of those who make reference in everydayness to conform those who are outside the standard, breaking imposed stereotypes, ready to reduce segregation with their struggle, promoting social mobility, fighting to eliminate the wage gap, when they dare to study careers with an enrollment with a predominantly man. Women who study Mechatronics Engineering and Electromechanical Engineering are a clear example of this. Investigating these students allows us to generate elements of self-reflection, when they mention, "I can do it, I can study this career that they say is difficult and is for men". The manifestations of stereotypes for women engineering students are a goal and a turning point to change the conceptions pre-established by their community. They are seen as Man, they are not able to do what men do, are ugly, messy, nerds, male tendency; these women are visualized each other as women who put on makeup, wear dresses, heels, go to the party, they are smart and able of doing what they want like any human being, no matter what the others say, desert's woman braking serotypes in engineering
\end{abstract}

\section{Keywords}

Women, study gender, stereotypes, engineering.

\section{Introducción}

Uno de los componentes claves para el desarrollo de un país, es el nivel educativo de su población, la cual está integrada por mujeres y hombres; en las últimas décadas se observa que en el nivel universitario, existe un incremento en la matrícula de las mujeres en ciertas áreas. Existen carreras con presencia predominantemente de los hombres, así como carreras con predominancia de mujeres, aun cuando existe una apertura en la educación superior en una aparente igualdad de género. Existe una elección simbólica de profesiones por género; el desarrollo laboral que implican y la percepción de los estereotipos vinculados a las capacidades de cada género. 
Los cambios sociales, incluida la discriminación que impedía la autonomía moral de las mujeres y el ejercicio de su libertad, han sido parte de la democratización en la actualidad y el compromiso del feminismo en la lucha por la igualdad. "Tras varios siglos de reivindicaciones de los movimientos feministas, la mayoría de los ordenamientos jurídicos de los Estados democráticos, recogen normas que establecen la igualdad formal de derechos entre todos los seres humanos. Pero a pesar de ese reconocimiento de la igualdad y de los derechos de las mujeres, lo cierto es que la lucha por la liberación femenina es una tarea inacabada: todavía perduran enormes diferencias entre los sexos tanto en el ámbito público como en el privado, las mujeres aún son discriminadas y en su vida cotidiana soportan numerosas desigualdades respecto de los hombres" Heras (2009).

Al consultar investigaciones de Martin Aurelia, 2006; Walsh Catherine, 2013; Miranda, 2007 y Davis Ángela, 2004; referidas al estudio de las mujeres, se identifican puntos esenciales que muestran, en primer lugar la desigualdad, enunciadas como: el segundo sexo; la segregación, los estereotipos, la violencia simbólica, la brecha salarial, la identidad. Las mujeres presentan desigualdad, aun entre distintas disparidades, como: las clases, las etnias, el color, el lenguaje, que coexisten a la par con los hombres. Uno de los caminos para disminuir la brecha de la desigualdad es la educación, no solo la formal, también la educación popular.

La experiencia y la ideología particular de las mujeres en la selección de sus estudios universitarios, que van ligados al desarrollo profesional, constituyen una fuente para comprender, desde otro ángulo, las razones de la limitada participación en la inserción de las mujeres a nivel licenciatura en las diversas carreras universitarias, con presencia predominantemente de hombres que ofertan instituciones de educación superior como lo es el Instituto Tecnológico de Cd. Jiménez (ITCdJ).

El Tecnológico Nacional de México es un sistema que brinda servicios educativos a nivel superior en las áreas de ingeniería y tecnología, cuenta actualmente con 260 Institutos Tecnológicos en toda la República Mexicana de los cuales 126 son Federales y 134 son Descentralizados, que permiten a 521,105 estudiantes a nivel licenciatura y posgrado, estar insertos en sus aulas en forma presencial o en la modalidad de distancia.

El Instituto Tecnológico de Ciudad Jiménez está ubicado en el sur del estado de Chihuahua, en la actualidad cuenta con una matrícula estudiantil propia del municipio y de varios municipios rurales en su colindancia, tiene una presencia de 23 años dedicada a formar profesionistas, en su mayoría en las áreas de ingeniería, contando con una carrera adicional de Contador 
Público. En el 2016, en el Tecnológico de Jiménez, el 40\% de su inscripción, eran mujeres.

A pesar del incremento gradual de la presencia femenina en la educación superior, existen aún rezagos de la inserción de las mujeres a nivel universitario.

La explicación de este hecho, según Norma Blazquez Graf. (2008), involucra dos elementos. Por una parte, la discriminación que aún existe en el seno de las comunidades científicas y, por otra parte, un fenómeno de autoexclusión determinado por la incompatibilidad, entre la vida familiar y social determinada por la asignación de roles y las exigencias de las instituciones científicas que favorecen marcadamente a los hombres.

En México, es esencial la incorporación de la perspectiva de género, prioritariamente en las instituciones educativas, ya que en ellas se realizan actividades para contribuir a la solución de las necesidades nacionales, coadyuvando a eliminar las persistentes desigualdades entre hombres y mujeres en el acceso a su producción y gestión, que inicia con la elección de estudios universitarios.

La educación formal de las personas en México, reproduce los estereotipos de género que continúan perpetuando el sometimiento de las mujeres, en un patriarcado eterno. Las relaciones de poder son evidentes, son latentes y se reproducen casi en forma automática.

Es importante revalorizar el papel que tiene la educación como formadora de sujetos sociales y de buscar el equilibrio entre la participación y las diferencias de hombres y mujeres.

Miranda (2007) hace visible que los estudios feministas relativos a la educación, enfatizaban no solo el papel de la educación en la reproducción de las clases y grupos sociales, sino también en la perpetuación del patriarcado. Si bien, las mujeres han alcanzado ya el $50 \%$ en casi todos los niveles educativos, tanto en la formación técnica como en la enseñanza superior, siguen dirigiéndose prioritariamente a ciertas áreas con mayor tradición femenina. Este autor realiza una investigación con datos longitudinales estadísticos, información bibliográfica y puntualizaciones concretas que viven las mujeres en Centro Universitario de Ciencias Económico Administrativas (CUCEA) de la Universidad de Guadalajara. El autor concluye que las estudiantes de clase media han conquistado espacios de igualdad, aun cuando se presenten barreras como el techo de cristal. La elección de carrera de las mujeres se da en profesiones que implican estereotipos de género y estas son desvalorizadas, por lo que es más complejo acceder al mundo 
laboral, desarrollarse en este y los salarios son bajos comparados con otras profesiones.

Una entrevista indagatoria de las mujeres en la educación superior, tanto a nivel licenciatura, posgrado e investigadoras científicas en el Ecuador, reconoce la existencia de una estructura androcéntrica en la ciencia y en las instituciones, en las entrevistas permea la percepción de que las mujeres luchan por la valoración de sus ideas, debido a que se adjudican el pensamiento abstracto a los hombres (Pessina, 2015).

La investigación emana de la observación de las composiciones de los grupos de asignaturas de las carreras universitarias donde se centran mujeres en ciertas licenciaturas y hombres en otras.

La perspectiva de género es el marco teórico en el que se basará el trabajo investigativo, ya que es un elemento con la potencialidad de reconstruir las concepciones históricas, sociales y filosóficas de la ciencia. Constituye un cambio significativo sobre el gran tema de la participación de las mujeres, como estudiantes de educación superior, pues permite explorar si en esta inclusión, intervienen elementos como los valores y esquemas socioculturales de género, y amplía el espectro de factores psicosociales y culturales que pueden contribuir a entender y redefinir los propósitos y metas de las mujeres en la educación superior, obteniendo así nuevos elementos de análisis y métodos diferentes para entender la realidad.

En un inicio de este estudio, se presentó la distribución de la matrícula en el Instituto Tecnológico de Cd. Jiménez, donde se muestra que las carreras con una presencia mayor de hombres es en Ingeniería Mecatrónica con un 92\% e Ingeniería Electromecánica con un 89.4\%. (Jiménez, 2016). En una segunda etapa se analizan las historias de vida de 14 mujeres universitarias, donde uno de los elementos centrales que se visualizan en este momento, es la percepción de estereotipos.

\begin{tabular}{|l|c|c|}
\cline { 2 - 3 } \multicolumn{1}{c|}{} & \multicolumn{2}{|c|}{2016 Enero - Junio } \\
\cline { 2 - 3 } & Hombres & Mujeres \\
\hline Ingeniería Mecatrónica (IM) & $93 \%$ & $8 \%$ \\
\hline Ingeniería Electromecánica (IE) & $89 \%$ & $11 \%$ \\
\hline Ingeniería en Sistemas Computa & $71 \%$ & $29 \%$ \\
\hline Ingeniería Industrial (II) & $65 \%$ & $35 \%$ \\
\hline Ingeniería en Gestión Empresaria & $44 \%$ & $56 \%$ \\
\hline Contador Público (CP) & $38 \%$ & $62 \%$ \\
\hline Porcentaje de Participación & $60 \%$ & $40 \%$ \\
\hline
\end{tabular}

Tabla 1. Matricula en porcentajes de Enero 2016, ITCdJ

Elaboración propia. 
Al centro de los cuestionamientos en esta investigación se ubica: ¿Qué características tienen las alumnas que optan por estudiar en carreras con presencia predominantemente de hombres en el Instituto Tecnológico de Cd. Jiménez? Por lo que la percepción incide en la participación que cada una de ellas realice y permita reflejarse en sus modos de actuación.

\section{Preguntas del avance de investigación}

- ¿En qué carreras tienden a situarse las mujeres en esta institución de educación superior tecnológica?

- ¿Consideran las mujeres que existen carreras exclusivas para los hombres?

- ¿Cuáles son los estereotipos de género que perciben las mujeres que estudian las carreras con presencia mayoritariamente de hombres en el ITCdJ?

- ¿Cómo se manifiestan los estereotipos de género en la alumnas que estudian las carreras con presencia predominantemente de hombres en el ITCdJ?

\section{Objetivo general}

Describir, analizar y reflexionar sobre las características de la participación de las alumnas que ingresan a estudiar a las carreras con matricula predominantemente de hombres, en el ITCdJ.

Se plantea un diseño metodológico con énfasis cualitativo, basado en un diseño narrativo donde se pretende analizar la sucesión de hechos, situaciones, fenómenos, procesos y eventos donde se involucran pensamientos, sentimientos, emociones e interacciones, a través de las vivencias de catorce mujeres universitarias quienes nos cuentan sus experiencias.

La fuente primaria de la investigación son las historias de vida, mediante la narrativa biográfica de catorce mujeres estudiantes a nivel licenciatura, del Instituto Tecnológico de $\mathrm{Cd}$. Jiménez, que se utilizaron como recurso metodológico. Se utilizó el paquete estadístico para las ciencias sociales (SPSS), para realizar el análisis de conceptos asociados.

Se contactó a cada una de las mujeres que formaban parte de estas carreras, con el fin de realizar una entrevista a profundidad, uno de los segmentos importantes de estas entrevistas fue cuando se habló de los estereotipos que ellas perciben directamente o que se reflejan en sus relaciones personales, como un elemento relevante de la socialización que experimentan por su género. 
El género, hace referencia a las diferencias socioculturales entre hombres y mujeres construidas sobre la base biológica. Es decir, mientras el término sexo se refiere a "las diferencias biológicas entre hombres y mujeres", género alude a roles, expectativas, funciones, valores, ... que cada sociedad adjudica a los sexos y que los seres humanos aprendemos y hacemos propios. Ruiz (2009).

Cada una de las estudiantes se presentó a su entrevista, antes o después de sus horarios de clases en el Tecnológico, robándole un tiempo al descanso, mostrando un compromiso con su tarea estudiantil.

En las entrevistas ellas coinciden en que no existen carreras exclusivas de algún sexo, es cierto que algunas carreras tienen una presencia mayoritaria de hombres o mujeres, este hecho no implica que sean exclusivas. En las carreras donde ellas interactúan perciben que las mujeres no se matriculan por un elemento referido a la autoconfianza de las mujeres, que es notoria por los comentarios en dos sentidos, uno de que es difícil y no pueden, el otro es el que dirán, dejándose influenciar por terceros. Las estudiantes de las carreras del ITCdJ reconocen la existencia de sus puntos débiles y de las fortalezas que las llevarán a lograr la meta de su titulación.

En los resultados del análisis de las entrevistas, algunos de los estereotipos que las estudiantes realizan son vinculados con la edad de las personas, una de ellas menciona:

"Cuando me preguntan que estás estudiando y les digo mecatrónica, me dicen: está muy difícil, es casi de hombres, esos comentarios son de personas ya más mayores, porque por ejemplo, empleados de la misma maquila donde trabaja mi mamá, les comenta que estoy estudiando eso, dicen: que padre pero son personas como que más jóvenes y con más mente abierta."

Otras reflexionan en que se les percibe como hombres, por lo que se imaginan como feas, desarregladas, nerds, situando a sus compañeras de carrera en otra apreciación distinta como menciona una estudiante de mecatrónica:

"Si, existen estereotipos que son como mujeres que no son arregladas, mujeres que son así más varoniles, no sé cómo explicarle, eh visto memes, me acuerdo de memes de mujeres que las ponen con bigotes, y cosas así. Mis compañeras son como cualquier otra mujer que pueda estar estudiando administración o derecho, son arregladas, pues yo las he visto y son arregladas, les gusta maquillarse, se ponen tacones y vestidos."

En las observaciones de su narrativa mencionan que no les importa el qué dirán, referente a lo que estudian, pues es lo que les gusta y quieren, a pesar de que otros digan que es difícil y de hombres, como menciona una estudiante: 
RECIE. Revista Electrónica Científica de Investigación Educativa Vol. 4, núm. 2, enero-diciembre 2019, pp. 1281-1290.

"Ah dije bueno, de que entre, la hago, la hago".

Al procesar su narrativa se detectaron un listado de palabras relacionadas con el concepto de estereotipo, se identificaron siete términos que conforman el diccionario de palabras que se presentan en la tabla No. 2 .

\begin{tabular}{|l|c|c|}
\hline Términos asociados a Estereotipos & Frecuencia & Porcentaje \\
\hline Bato & 2 & $7 \%$ \\
\hline Hombre & 9 & $32 \%$ \\
\hline Varonil- Marimacha & 2 & $7 \%$ \\
\hline Feas & 3 & $11 \%$ \\
\hline Desarregladas & 2 & $7 \%$ \\
\hline $\begin{array}{l}\text { Inteligentes (Nerds) } \\
\text { No pueden hacer cosas como los }\end{array}$ & 2 & $7 \%$ \\
\hline Total & 28 & $29 \%$ \\
\hline
\end{tabular}

Tabla No. 2 Diccionario de conceptos asociados sobre Estereotipos. Elaboración propia

De acuerdo al diccionario de conceptos asociados, es posible observar que casi una tercera parte de las estudiantes asocia a los estereotipos únicamente con el termino Hombre, esto es, que se perciben como parte de la predominancia de los hombres; un poco más de una cuarta parte de las alumnas perciben que se les ve como que No pueden hacer cosas como los hombres.

Entre el término intermedio (de acuerdo al número de menciones) se identifican "Feas" como palabra que el 11\% de las discentes de las carreras de electromecánica y mecatrónica asocian a los estereotipos que les son impuestos.

Como términos con menor número de menciones, podemos ubicar a la percepción de "Bato" con un 7\%, que es una percepción de Hombre en forma despectiva, junto con que se les percibe como "Marimachas-Varonil", con el mismo porcentaje se designa como "Desarregladas", con este mismo puntaje con una connotación positiva el ser "Inteligentes" (Nerds) aunque para algunos se perciba como matadas en estudiar.

Para profundizar con el análisis de datos, se realizó una codificación de los términos que conforman el diccionario de palabras; se procedió a generar una clasificación binaria (presencia o ausencias significadas por $1 \mathrm{y}$ 0 respectivamente) a partir de ello, se produce un dendrograma en el que 
quedan representadas las etapas del proceso de fusión y las distancias existentes entre los elementos fundidos en cada etapa, como se muestra en la Figura No. 1. En los datos procesados dio por resultado de .78 la desviación estándar.

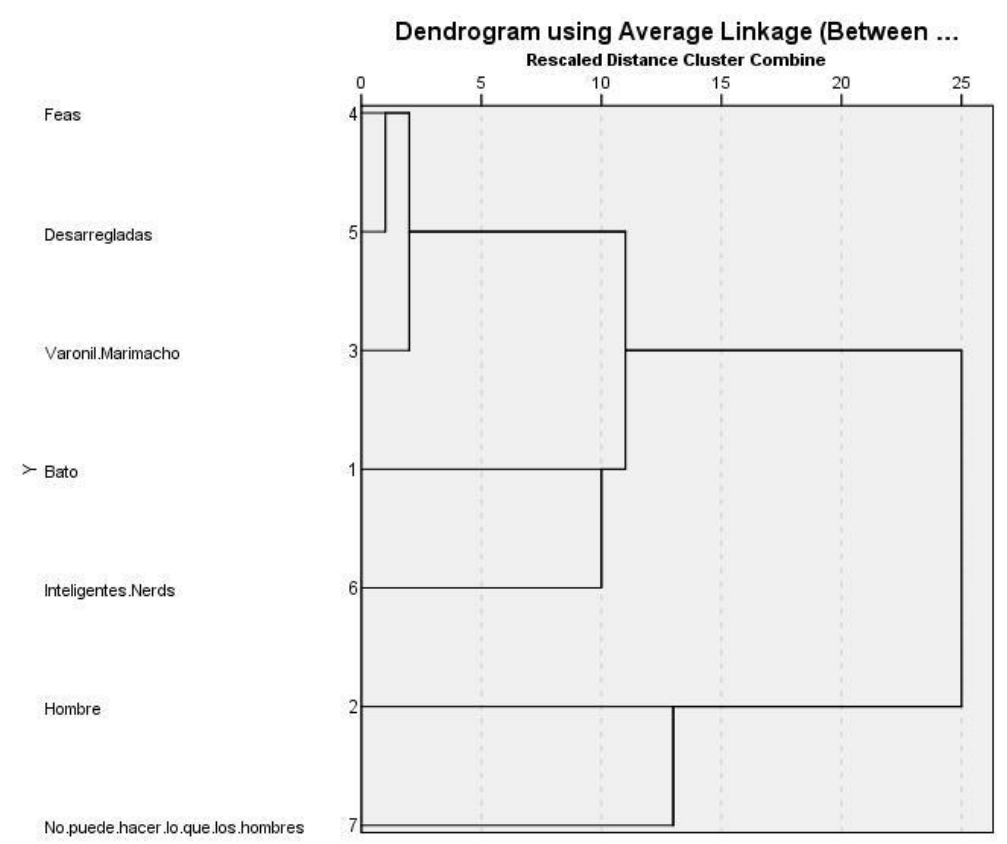

Figura No.1 Campos semánticos sobre los estereotipos.

Se puede observar como las estudiantes manifiestan que los estereotipos concebidos son que se les percibe como hombres, como un bato más, de forma varonil (marimacho).

Conocer los casos de éxito de las mujeres en las aulas universitarias, le puede brindar a otras mujeres la oportunidad de fortalecer sus decisiones escolares y lograr un título universitario que les permita una movilidad social esperada. La confianza en sí mismas les permite a las mujeres ir reflexionando sobre su contexto y cambiar sus prácticas, esto incluye la presencia y elección de carrera que determina para cimentar su vida laboral (Jiménez, 2016).

Las estudiantes de Ingeniería en Mecatrónica e Ingeniería Electromecánica, perciben que existen estereotipos que se manifiestan no solo en los comentarios de la familia, los compañeros, la sociedad, en algunos casos en el acceso de la utilización de herramientas por parte de algunos compañeros, lo que requiere la búsqueda de distintas fuentes para el aprendizaje de las habilidades requeridas en el uso de maquinaria y 
herramienta, una estudiante comento, "tal vez no tenga la fuerza de un hombre, pero sé utilizar herramienta que me permite realizar las maniobras y lograr la practica requerida, con ello hacer un buen trabajo". Están convencidas de que la carrera elegida para desempeñarse es lo que quieren y donde pueden utilizar sus habilidades y destrezas, son parte de un reto, ahora estudiando y después trabajando, conocedoras de que son capaces de conquistar la meta que se tracen, porque son capaces de romper con los estereotipos establecidos en este desierto del sur de Chihuahua.

\section{Referencias}

Davis, Angela. Y. (2004). Mujeres, raza y clase (Vol. 30). Ediciones Akal.

Graf, N. B. (2008). El retorno de las brujas: incorporación, aportaciones y críticas de las mujeres a la ciencia. Universidad Nacional Autónoma de México, Centro de Investigaciones Interdisciplinarias en Ciencias y Humanidades.

Guevara, Patricia. G. (2002). Las carreras en ingeniería en el marco de la globalización: una perspectiva de género. Revista Latinoamericana de Estudios Educativos (México), 32(3), 91-105.

Jiménez Hidalgo, Guadalupe (2016) Distribuciones de género en la matrícula de nivel superior de las carreras en el Instituto Tecnológico de Cd. Jiménez 2015-2016.

Las Heras Aguilera, S. (2009). Una aproximación a las teorías feministas.

Martín Casares, A. (2006). Antropología del género. Culturas, mitos y estereotipos sexuales. Feminismos. Madrid. Ediciones Cátedra.

Miranda Guerrero, R. (2007). Mujeres, educación superior e igualdad de género. CPU-e, Revista de Investigación Educativa, (4).

Papadópulos, J., \& Radakovich, R. (2006). Educación superior y género en América Latina y el Caribe. Sitio en Internet) Disponible en http//www. cse. cl Salazar, José Miguel (2005) Educación superior y género: Tendencias observadas.

Pessina Itriago, M. (2015). Mujer, conocimiento y ciencia (Master's thesis, Quito: FLACSO Sede Ecuador).

Ruiz, E. E. (2009). Estereotipos de género. Padres y Maestros/Journal of Parents and Teachers, (326), 17-21.

Walsh, Catherine. E. (Ed.). (2013). Pedagogías decoloniales: prácticas insurgentes de resistir,(re) existir y (re) vivir. Abya Yala. 